\title{
LA ILUSTRACION Y EL ROMANTICISMO COMO CUNA DEL IDEALISMO
}

\section{Miguela DOMINGo CENTENo}

«Se ha afirmado que el Idealismo Alemán es la teoría de la Revolución Francesa ${ }^{1}$ ". Ello es parcialmente cierto, pues una porción sustancial de la corriente filosófica que se ha llamado Idealismo Alemán consiste en la teorización sobre los cambios originados por la Revolución. Tanto el movimiento político francés como el filosófico germánico son los hechos más importantes que presenciaron las postrimerias del siglo $\mathrm{XVII}^{2}$. El Idealismo Alemán debe comprenderse habida cuenta de las particularidades de la Ilustración Germánica (en alemán, Aufklärung) y del Movimiento Romántico, cuyo ápice fue alcanzado por la misma Alemania.

La Ilustración llegó a Alemania, procedente de Francia y tuvo que ser asimilada por un país cuyo Renacimiento había sido un asunto más de reformadores religiosos que de humanistas con tendencias laicas. La Ilustración en Alemania se encontró con una atmósfera mucho menos secularizada que en Francia o que en la misma Inglaterra. Durante los años más brillantes de la Aufklärung esta coexistió con un intenso movimiento de misticismo pietista. La afectividad y el sentimiento cuasi religioso no le fueron del todo ajenos. Cuando Goethe ${ }^{3}$ y Herder $^{4}$ abandonaron el Racionalismo

${ }^{1}$ Enrique G. Arboleya: Historia de la estructura y del pensamiento social, Madrid,1975, Vol. I, p. 435.

${ }^{2}$ Lo que dio tal fuerza y vigencia al Idealismo Alemán fue precisamente el añadir a todo el saber racionalista de la Ilustración un elemento de toma de conciencia histórica y otro elemento de nacionalismo, ambos puestos en primer planopor la Revolución.

3 Aún cuando Goethe reconoció que no poseía "órgano apropiado" para el pensamiento filosófico, desarrolló ciertas ideas filosóficas inspiradas en la naturaleza y en el conocimiento de ciertos autores de tendencia más o menos "monista".

Éndoxa: Series Filosoficas, $n^{\circ} 7,1996$, UNED, Madrid:

Miguela Domingo Centeno: La llustración y el Romanticismo como cuna del idealismo. pp. 201-215. 
de la Ilustración para fundar la tendencia del Asalto y Empuje (Sturm und Drang) $)^{5}$, con ello, el Romanticismo, el paso parecía del todo natural. La preeminencia de la afectividad en el pensamiento alemán de la época que vio surgir y desarrollarse el Idealismo, va unida también con la situación especial de la nación germánica. El espíritu de la llustración del siglo XVIII se extrajo de la Revolución Científica e Intelectual del siglo XVII. La Ilustración transmitió (y popularizó) la filosofía de la ley natural y del derecho natural. Nunca hubo una época tan escéptica respecto a la tradición, tan confiada en los poderes de la razón humana y de la ciencia, tan firmemente convencida de la regularidad y armonía de la naturaleza, y tan profundamente imbuida del sentido del avance de la civilización y del progreso. Los pensadores de la época creían en la unidad de la humanidad y sostenían que todos los hombres vivían bajo la misma ley natural del derecho y de la razón. Suponian que todos los hombres participarian igualmente en el progreso y que, a largo plazo, todos ellos estarían de acuerdo, y que el resultado de la historia sería una civilización uniforme, en la que todos los pueblos y razas participarían en igual medida.

${ }^{4}$ Su influjo en la formación del Sturm und Drang fue decisivo. La importancia de Herder estriba, nada menos, que en ser el padre del historicismo, de la noción de Volkgeist y del nuevo nacionalismo romántico.

Herder fue el que inició la reacción contra el racionalismo de la Ilustración (Ver Berlin Isaiah: J. G. Herder, ensayo aparecido en Ecounter, julio, 1965, Vol. XXV, ${ }^{2}$ 1 , p. 29 y siguientes). Sostuvo que cada actividad y circunstancia humana, cada civilización, tenía una cualidad singular, que no podía reducirse a regularidades universales y a leyes cuantitativas, lo cual contrastaba con la creencia en leyes inalterables y universales, aplicables también al hombre y a su historia, típica de la Aufklärung y la Ilustración Francesa.

Todo esto no quiere decir, como algunos críticos han creído, que Herder fuera un antirracionalista a ultranza. Ni su nacionalismo permite tampoco que se le tilde de chauvinista. Precisamente Herder, como Goethe, es un nacionalista cosmopolita, un historicista respetuoso de la ciencia positiva. Herder percibe los diversos niveles en los cuales se mueve la conciencia humana, y no permite que el intuitivo o emocional, desbanque al racional, o viceversa.

${ }^{5}$ Tempestad $e$ ímpetu, del título de un drama de Maximiliano Klinger de 1776. Es la expresión filosófica del movimiento literario-político que luchaba contra la razón finita. 
Todo el pensamiento de la época, se proponía hacer a todos los hombres libres. Todo el pensamiento de la llustración, de un modo u otro, estaba relacionado con el problema de la libertad . $^{6}$.

Pero fueron los años de la Revolución Francesa los años de máximo florecimiento cultural para Alemania. Las ideas alemanas coincidieron con todo el fermento del pensamiento social conocido como "Romanticismo", que en todas partes se enfrentaba con las "secas abstracciones" de la Edad de la Razón. Alemania se convirtió en el más romántico de los países, y la influencia alemana se extendió por toda Europa.

Con el término "Romanticismo" que al comienzo se refería a la novela caballeresca, rica en aventuras y amores, se indica el movimiento filosófico, literario y artístico que se inició en Alemania en los últimos años del siglo XVIII, alcanzó su máximo florecimiento en toda Europa durante los primeros decenios del siglo XIX y constituyó la nota característica de este siglo.

El significado corriente del término "Romanticismo", que equivale a sentimental, deriva de uno de los aspectos más llamativos del movimiento romántico, del reconocimiento del valor por él mismo atribuido al sentimiento: una categoria espiritual que la antigüedad clásica había ignorado o despreciado, que el Iluminismo del siglo XVIII había reconocido en su fuerza y que en el Romanticismo adquiere valor predominante. Este valor predominante es la herencia principal que el Romanticismo recibe del movimiento Sturm und Drang; es aquí donde la razón continuaba siendo para los seguidores del Sturm und Drang, lo que era para el Iluminismo: una fuerza finita capaz ciertamente de transformar

${ }^{6}$ El Iluminismo Alemán debe su originalidad, respecto al inglés y francés, más que a nuevos problemas o temas especulativos, a la forma lógica en que los temas y problemas se presentan y manejan. El ideal de una razón que tenga el derecho de atacar con sus dudas y sus problemas todo el mundo de la realidad, se transforma en la hustración Germánica en un método de análisis racional, a la vez cauto y decidido, que avanza demostrando la legitimidad de cada paso y la posibilidad intrínseca de los conceptos de que se sirve y su fundamento (Grund). Es este el método de la fundamentación que debía ser caracteristico de la filosofía alemana posterior. 
gradualmente el mundo, pero no absoluta ni omnipotente $y$, por lo mismo, siempre más o menos en oposición con el mismo mundo y en lucha con la realidad que dicha fuerza estaba destinada a transformar.

Por su parte, el Romanticismo nace cuando este concepto de la razón es abandonado y se comienza a entender por razón una fuerza infinita que habita en el mundo y lo domina, constituyendo así la sustancia misma del mundo; este cambio lo efectuó Fichte, que identificó la razón con el Yo infinito o Autoconsciencia absoluta, e hizo de ella la fuerza por la cual el mundo entero es producido: «Nos proponemos escribe Fichte en la primera página de su Teoría de la Ciencia - investigar el principio absolutamente primero, absolutamente incondicional de todo saber humano. Si este principio debe ser absolutamente primero, no tolerará que se le demuestre, ni que se le defina (beweisen oder bestimmen). Debe expresar una acción (Thathandlung) no sujeta, de hecho ni de derecho, a ninguna determinación empírica de nuestra conciencia, por lo mismo que esa acción es el fundamento y la condición misma de posibilidad de toda conciencia»?

Pero sobre el fundamento común de estos caracteres, los Románticos interpretaron el Principio infinito de dos maneras fundamentalmente distintas:

1- La primera interpretación, más próxima a las ideas del Sturm und Drang, considera el infinito como sentimiento, o sea, como actividad libre, carente de determinaciones o más allá de toda determinación.

2- La segunda interpretación entendía el infinito comó Razón absoluta que se mueve con necesidad rigurosa de una a otra determinación, de modo que cada determinación puede deducirse de la otra necesariamente.

Lo Absoluto que se exige para la racionalidad del objeto (pensado) no puede buscarse en un objeto opuesto al sujeto 91.

${ }^{7}$ Sämmtliche Werke, Grundlage der gesammten Wissenschaftslehre, Band I, 1794, p. 
trascendental, sino que debe colocarse en este sujeto mismo, que, por lo tanto, se pone como actividad absoluta. Consiguientemente, el objeto, tanto en su contenido fenoménico (material), como en su forma de objeto, debe explicarse racionalmente por el sujeto. De ahí el Monismo ${ }^{8}$ absoluto del sujeto y la inmanencia total del objeto en el sujeto. El esfuerzo del Idealismo Absoluto recaerá principalmente en la derivación racional, sistemáticamente de todo el objeto a partir del sujeto absoluto.

Pero la significación de los grandes sistemas idealistas no se agota en lo que tienen de sistemático; ve en ellos el desarrollo de un capital filosófico que, en cuanto filosófico, no es idealista, sino que es, o debiera de ser, peculiar de toda filosofía. El interés dominante no radica en las grandiosas doctrinas conforme al punto de vista parcial de los grandes maestros, sino en la vastedad del horizonte de problemas y en el rigor de su penetración.

La serie de pensadores filosóficos que llamados "Idealistas Alemanes", es decir, la pleamar, única en su género, de sistemas que se agolpan y se superan, y el riquísimo encadenamiento de controversias literarias, cuyo conjunto representa para la posteridad la "época del Idealismo Alemán", es un movimiento espiritual que, en cuanto a concentración y altura especulativa, casi ningún otro puede ponérsele a la par.

Lo que reúne estrechamente en un grupo unitario a los pensadores del Idealismo Alemán, no obstante de su profunda diferencia y aún sus conscientes oposiciones y diferendos, es, en primer lugar, la común situación del problema. Para todos ellos el punto de partida es la filosofía kantiana, cuya riqueza inagotable

${ }^{8}$ Es caracteristico del monismo reducir cualquier sustancia a la que se estima como única existente, declarando que o bien no existe, o bien es únicamente una mera apariencia de la substancia, o del tipo de substancia existente.

El monismo del que aquí hablamos, es un monismo gnoseológico, en el cual la realidad a la cual el monista reduce cualquier "otra" es el sujeto. La misión de la gnoseología es "de apprehensione cognoscibilis \& principiis essendi agens". En alemán se usa con mayor frecuencia "Erkenntnistheorie". 
en los planteamientos de los problemas, siempre suscita renovados intentos de solución.

La meta común a todos es la creación de un vasto sistema de filosofia, estrictamente unitario y basado en fundamentos últimos e inconmovibles. A todos se les presenta claramente ante los ojos el ideal de aquella "metafísica futura", a la que la denodada labor intelectual de Kant sólo había aportado Los Prolegómenos. Por cierto, no se les escapaba que Kant en las dos últimas Criticas había instituido esta metafísica en sus lineas fundamentales. Pero a ellos no les bastaban dichas lineas fundamentales. De un sólo golpe y a través de una unívoca certeza debe nacer el sistema que llevará a término la idea de la filosofía.

La dirección en que buscaban este sistema ideal difiere en cada uno, y así, de hecho, en cada nuevo proyecto se gesta un sistema diferente. Pero a todos ellos es común la creencia de que semejante ideal es posible, e inclusive alcanzable por la razón humana. Todo el movimiento se halla bajo el signo de un optimismo filosófico de vigor juvenil y propenso a crear. Para estos pensadores todo escepticismo significa un estadio de tránsito, es decir, una instancia de prueba y de reflexión, $o$ bien un camino que lleva a una interiorización y agotamiento de los problemas.

Los primeros pensadores postkantianos se proponen no tanto transformar como comprender la verdadera doctrina kantiana. El hecho de que no abundara semejante comprensión no ha de sorprender, dada la dificultad de las investigaciones de La Critica de la Razón Pura.

La filosofia popular que predomina en la época de la Mustración expirante no había adelantado en esta tarea. Lo que el "sano entendimiento humano" no podía concebir, tenía que juzgarlo como paradójico, y hasta como amenazador de su propia autoridad. Cuanto menos se entendía a Kant, tanto más absurda tenía que parecerle a cualquiera la empresa de La Crítica; hasta los pensadores más serios de la Escuela Wolffiana, como Moses Mendelssohn, sólo supieron sacar de ella el aspecto negativo, es decir, el escepticismo metafísico. En su obra Popularphilosoph der 
deutschen Aufkärung, Mendelssohn nos dice «que de la simplicidad del alma, se deriva la imposibilidad de ser aniquilada, ya sea instantáneamente, ya sea gradualmente», opinión a la cual se opuso Kant en la sección de La Crítica de la Razón Pura que trata de la «Refutación de la prueba de Mendelssohn de la permanencia del alma»?.

De un modo semejante la juzgó Herder, discípulo de Kant en su período precrítico, intentando mostrar en su Metacrítica, que el origen del conocimiento radica en las sensaciones del alma y en las analogías que ésta establece a base de las experiencias de sí misma. Las categorias no son, pues, según Herder, nociones trascendentales, sino resultados de la organización de la vida.

Fichte lleva a cabo, en el terreno del Idealismo, lo que Spino$\mathrm{za}^{10}$ había intentado en vano realizar en el campo del Realismo. Su sistema es el "spinozismo invertido" y, gracias a esta inversión, un "spinozismo transfigurado": es la fundamentación y el desarrollo completo del principio general de toda especulación, sin el que este principio se confundiría, como en Spinoza, con un ser absoluto dogmáticamente afirmado. La comprensión - tal es el punto angular del pensamiento de Fichte-no puede lograrse nunca más que por medio de una acción y una creación ideal: «el hombre sólo llega a comprender en cuanto -convirtiendo la cosa en simple forma - hace de la forma cosa, y reduce la cosa a la nada. Sólo comprendemos una cosa, cuando podemos construirla, hacerla nacer, surgir en pensamiento ante nosotros. Cuando no podemos construirla, hacerla brotar por nosotros mismos en el

9 Pedro Ribas: Crítica de la razón Pura, edic. Alfaguara, Madrid, 1978, B 414-B 426.

${ }^{10}$ Aparte de Lessing, Jacobi fue el primero que supo comprender verdaderamente el genio filosófico de Spinoza, aunque no se cansara de poner de relieve y de combatir los peligros de sus doctrinas metafísicas fundamentales (Ver Ueber die Lehre des Spinoza in Briefen an Herrn Moses Mendelsohn en F. H. Jacobi, Briefwechsel, ed. frommann-holzboog, Stuttgart, 1981/89, Reihe I, Band 2, en las que Jacobi comunica los coloquios que habia mantenido con Lessing el 7-8 de julio de 1780 , en los que Lessing manifestaba su adhesión al spinozismo). 
pensamiento, es que no la comprendemos. Por lo tanto, si un ser ha de convertirse en un objeto plenamente comprendido por nosotros, tenemos que anularlo, destruirlo objetivamente -como existente de suyo- en el pensamtento, para hacer que surja de un modo puramente subjetivo, como nuestra propia creación, convertido en un simple esquema. No debe quedar en él, formando parte esencial de su concepto, nada que no sea obra propia nuestra, que no sea, ahora, una simple proyección de nuestra imaginación creadora. Querer formarse un concepto del ser equivale, por tanto, a destruirlo como ser sustantivo, independiente de nosotros. $Y$ lo que queda en pie para nosotros, después de esa destrucción, no es otra cosa que la misma subjetividad vacía, constantemente renovada y repetida hasta el infinito. En el fondo, sólo existe para nosotros un conocimiento: no el del objeto, sino el del mismo conocer, una acción simplemente de la acción y en función de ella; un acto que sólo se tiene presente y se considera a si mismo, simplemente en gracia al mismo acto y a su consideración, sin otro sujeto ni objeto; sin "en", "de", "para" ni "a"»".

El principio de identidad, la fórmula $a=a$ es, en realidad, la expresión metodológica adecuada y el punto de partida exacto de esta teoría. Pero cabe, naturalmente, preguntarse: ¿cómo puede haber, dentro de este modo de consideración, un " $a$ " comparable consigo mismo y equiparable a sí mismo? Comprendemos perfectamente la relación, comprendemos la necesidad de la ecuación misma; pero, ¿de dónde sale, no la relación, sino lo relacionado?. A esta pregunta no puede contestar, en modo alguno, La Teoría de la Ciencia ni el Idealismo Trascendental. Lo que éste nos ayuda a comprender, lo que nos promete poner al descubierto, y nos descubre, en efecto, hasta en sus últimas condiciones fundamentales, es la forma del pensamiento; pero todo pensamiento de un algo, representa para ella un problema insoluble. La imaginación trascendental, a la que se nos remite para que encontremos en ella el origen de la representación del

11 Jacobi a Fichte, 1799 (Ibid., p. 9-57). 
objeto, del "objeto" del conocimiento, no puede, manifiestamente, ayudarnos en nada, desde este punto de vista: «ella misma no es, al fin y al cabo, otra cosa que una expresión psicológica de la misma actividad pura y simple del relacionar unas cosas con otras, un ir y venir en el reino de la pura fantasía, sin aquí ni allí, sin intención ni penetración, un simple poetizar, una mera actuosidad en el campo de la pura conciencias ${ }^{12}$.

Pero fue Karl Leonhard Reinhold el que rechazó de Fichte el activismo absoluto de la conciencia, elaborando una filosofía de la unidad, basada en la idea de representación, y no en una intuición intelectual.

Unificó la sensibilidad, el entendimiento y la razón por medio del reconocimiento de lo que consideraba un "hecho primario", es decir, la "facultad representativa" o "representación". Tal facultad fue considerada por Reinhold como distinta de la sensibilidad, del entendimiento y de la razón, pero a la vez como el fundamento de cualesquiera y de todas ellas, ya que en la representación se encuentra lo que representa -el sujeto- y lo representado -el objeto-, así como la tendencia a la voluntad, a lo sensible y su obediencia al imperativo moral. Estas reflexiones de Reinhold culminaron en su "filosofía elemental" y en lo que llamó "el principio de la conciencia", es decir, el principio según el cual «la representación de lo que representa y lo representado, se hacen en la conciencia distintos y a la vez relativos al ser consciente».

Es mérito de Reinhold el haber dado el impulso más decisivo para promover una diferente forma de estimación de la filosofía kantiana. Dieron la iniciativa sus Briefe über die kantische Philosophie ${ }^{13}$ publicadas en el Deustscher Mercurde Wieland; con una acertada decisión, copiando de un modo fiel el camino que él

12 Ueber das Unternehmen des Kritizismus die Vernunft zu Verstande zu bringen (Ibid., p. 99).

13 Karl Leonhard Reinhold: Korrespondenzausgabe, 6 Bände, ed. frommannholzboog, Stuttgart, 1983, Band I (Correspondencia desde 1773-1788). 
mismo se había abierto para la inteligencia de La Crítica de la Razón Pura, convirtió en punto de partida aquellos aspectos de la doctrina kantiana que generalmente se resistian a la comprensión de un círculo más amplio: a saber, los problemas morales y religiosos.

Sin embargo, con la divulgación de la doctrina kantiana no se introduce únicamente la interpretación de su sentido peculiar, sino casi al mismo tiempo la tendencia a rebasar algunos de sus puntos insatisfactorios. Reinhold mismo hizo el primer intento de este tipo, y así, al par, llegó a convertirse en el primer continuador de la nueva doctrina. El impulso que parte de él es, desde el comienzo, doble: por una parte va hasta Kant, pero, por otra, va más allá, es decir, acusa la tendencia a rebasarlo ${ }^{14}$. Ambas direcciones se reflejan con claridad en el desarrollo que arranca de él y, en parte, son las mismas cabezas filosóficas las que continúan trabajando tanto en una como en otra dirección. Sin embargo, los dos movimientos describen círculos muy diferentes que requieren una atención especial.

Inmediatamente vinculada a Reinhold surge en la última década del siglo XVIIl una serie de defensores y adversarios de la filosofia crítica, que en primer término se entregan a la interpretación de Kant y a tomar posición frente a él. A esta serie pertenecen Schulze, Maimon y Beck; emparentados de un modo más lejano están Jacobi y Bardili. Sólo pocos años después, y todavía en la

${ }^{14}$ En los círculos de los primeros discípulos y partidarios suyos, Reinhold pasa por ser, no sólo el más autorizado intérprete de las doctrinas kantianas, sino el filósofo que da verdadera solución a los problemas simplemente planteados por Kant.

Por el contrario, el Idealismo especulativo de Fichte y Schelling, considera a las doctrinas de Reinhold, en última instancia, pura y simplemente como una nueva versión de la antigua filosofía popular.

Finalmente, el juicio que la concepción moderna emite acerca de Reinhold y que puede considerarse casí como el juicio general, ve en él el primero y típico exponente de esa dirección del pensamiento caracterizado como la tergiversación psicologista de la filosofía crítica (Citado por José Ferrater Mora en Diccionario de Filosofia, ed. Alianza, Madrid, 1988, T. IV, p. 2822-2823). 
misma década, con la presencia de Fichte se inicia un movimiento nuevo y más vasto, cuyos conductores se asignan independientemente los más altos objetivos especulativos.

La temprana escuela de Poetas románticos desempeña un particular papel en este movimiento filosófico. Su influjo se ejerce casi contemporáneamente al de los primeros trabajos de Schelling y en estrecha relación recíproca con los progresos de este filósofo. Son F. Schlegel y Novalis, ante todo, los que se aventuran en el campo filosófico y consagran a la especulación idealista el sentido de su anhelo encaminado a lo infinito.

El Romanticismo está indisolublemente vinculado a la filosofía de Fichte y de la juventud de Schelling. El reino del alma en su particular autonomía, tal como Schlegel, Hölderlin y Novalis lo buscaron, pareció encontrar su precisa acuñación filosófica en la doctrina del Yo autónomo, creador de todo. Según Fichte toda espontaneidad reside en la imaginación productiva, y semejante espontaneidad es lo que concibieron los Románticos. La creación poética en toda circunstancia arraiga en ella y esta creación es el foco de interés del Romanticismo.

Friedrich Von Schlegel, gran figura de la literatura romántica alemana, reconoce en la doctrina de Fichte que ella nunca es meramente filosofía, sino, al par, filosofía de la filosofía. El pensamiento filosófico, por encima de todos los objetos particulares, inclusive por encima del universo, tanto material como espiritual, se pone a sí mismo como objeto. Y aquí está el punto en que se vuelve atrás, pues se vuelve hacia lo subjetivo. El filósofo mismo es también «un fenómeno filosófico interesante; mientras filosofa, es objeto de su propia burla» ${ }^{15}$, es decir, de su ironia.

Schlegel es totalmente sincero cuando considera a Fichte como el maestro de la burla, inclusive cuando tiene a $\mathrm{Kant}^{16}$ por burlón.

\footnotetext{
${ }^{15}$ La burla es la manifestación, es decir, el destello exterior de la fantasía.

${ }^{16}$ Hay que tener aquí presente el fragmento 220 del Atheneum que, entre otras cosas, dice: "sucede con las ideas como con las melodias preferidas. Los Kantianos canturreándolas (se entiende, las ideas de Kant) las han matado; razón por la cual fácilmente se le puede desconocer y tener por menos burlón de lo que es" (Ver Die
} 
La burla es una categoría sui generis propia de toda verdadera grandeza, y hasta un criterio de valor de lo grande. Existe para Schlegel un instinto original de la grandeza que llamamos moral. Quien no la tiene sólo comprende lo común, lo vulgar que es equivalente a su simpleza. «El hombre simple juzga a todos los hombres como hombres, pero los trata como cosas y no comprende en absoluto que aparte de él haya hombres diferentes ${ }^{17}$. Para Schlegel, la conciencia estética es la única que puede enlazar el mundo fenoménico con el nouménico, que habían sido separados por Kant y que se unificaron bajo la égida de los grandes idealistas postkantianos.

El artista, y especialmente el poeta, no tienen que someterse a los principios que se supone rigen el mundo, porque pueden transformar el mundo. En rigor, el poeta crea mundos y con ello expresa la libertad máxima. La formación del Romanticismo literario, en su derivación fichteana, se puede seguir claramente en la figura de Schlegel. En 1795, en las cartas a su hermano Augusto Guillermo, Schlegel se pronuncia de la manera más entusiástica por la doctrina de Fichte. Y en el epílogo del ensayo Sobre el estudio de la poesía griega, después de haber terminado tres periodos de la teoria estética: el primitivo, dominado por el principio de autoridad; el dogmático de la estética racional y empírica, y el crítico considera a Fichte como aquel que puede conducir a su cumplimiento la estética crítica; "puesto que Fichte -dice- ${ }^{18}$ ha descubierto el fundamento de la filosofia crítica, tenemos ya un principio seguro para rectificar, contemplar y llevar a término el plan kantiano de la filosofía práctica; y no está ya justificada la duda acerca de la posibilidad de un sistema objetivo de las ciencias estéticas, prácticas y teóricas". En realidad, el concepto de

\footnotetext{
Romantische Schule, Berlín, 1870).

17 Atheneum, fragmento 339 (Ibid.)

${ }^{18}$ Seine prosaischen Jugendschriften (Primeros escritos en prosa) en Briefe, $1^{\text {a edición, }}$ ed. Minor, Viena, 1882; 4" edición por O. Walzel, Berlín, 1920, p. 236-244.
} 
la poesía romántica, tal como es expresado por Schlegel ${ }^{19}$, no es otro que la transferencia al dominio de la poesía, considerada como un mundo en sí, del principio fichteano de lo infinito. La poesía romántica es la poesía infinita, universal y progresiva. Identificada con lo infinito, la poesía absorbe en sí todo el mundo y se apropia los objetivos que aparecen fragmentados y dispersos en los diversos aspectos de la cultura; «sólo ella es infinita, de la misma manera que sólo ella es libre y reconoce como su primera ley la siguiente: que el albedrío del poeta no soporta ley alguna $"{ }^{20}$. Lo que en Fichte era la libertad del principio infinito, para Schlegel es el albedrío absoluto del genio poético.

Por otro lado, consideremos la figura de Hölderlin, cuyos tempranos bosquejos poéticos muestran una callada lucha contra el espíritu de la filosofía de Fichte. Hölderlin no queda satisfecho ante la tesis de la Teoría de la Ciencia, de que la naturaleza sea en sí misma un vano producto cuya significación se agota en resistir a la actividad del yo práctico. El eticismo de Fichte le parece la negación y la difamación del mundo creado, cuyo esplendor está, para él, por encima de toda duda. Pero no ejerce una crítica ontológica, sino una crítica muy delicada inmanente. Si el destino de la naturaleza consiste en ser eliminada, como pensaba Fichte, el yo aniquila su No-yo, por tanto, elimina la relación con el polo opuesto en que consiste precisamente la relación ética; pues el yo práctico tiene que tener un mundo sobre el cual actúe, como lo demuestra la Doctrina de la Ciencia. El yo práctico se eliminaría a sí mismo. Para Hölderlin la naturaleza es más que el mundo exterior dado, es el elemento en el que el vive y respira, inclusive es un ser viviente que lo abraza con amor y lo sustenta, y justamente por eso es un ser real que se experimenta y se vive inmediatamente por encima de toda duda. Es para él un ser

19 Fue Schiller el que llevó a Schlegel a sus reflexiones filosóficas sobre la diferencia entre el espiritu clásico y el espíritu romántico, y sobre la preeminencia del último.

${ }^{20}$ Seine prosaischen..., Ibid., Kritische Fragmente 116. 
divino, colmado de divinidades, infinitamente más grande que el hombre ${ }^{21}$. No obstante, fue amigo de Schelling, de Hegel y admirador de Fichte. Su novela Hiperión ${ }^{22}$, en la que expresa sus ideales y sus convicciones filosóficas, contiene todos los rasgos de la concepción romántica, y está plagada de consideraciones filosóficas que revelan claramente el influjo de Fichte y Schelling.

También el tercero de los Románticos, F. Von $\mathrm{H}$. Novalis, parte de Fichte. Al pensamiento fundamental de la Doctrina de la Ciencia referente al yo como fundamento de toda existencia, corresponde en Novalis una inclinación natural a penetrar en el mundo maravilloso de la propia interioridad. Tiene a Fichte por descubridor de la ley interior del mundo, de lo que la exterior sólo es un reflejo. Y, lo mismo que Fichte, se encuentra en el fondo del yo, no con una capacidad entregada a la contemplación teórica, sino con una capacidad activa y práctica. La voluntad es la fuerza central del espíritu; en el sentimiento moral poseemos una capacidad creadora. La voluntad no es sólo libre, es también verdaderamente omnipotente: puede lo que quiere.

La Doctrina de la Ciencia es meramente "logología"; pero el yo es más que lo lógico. Es la patria verdadera de lo maravilloso en que nosotros originalmente estamos afincados, pero de la que incomprensiblemente nos hemos distanciado. Se necesita de una "psicología real" para orientarnos nuevamente en ella. Aquí se trata "de buscar nuevas fuerzas insospechadas y de rastrear las relaciones existentes entre ellas". Cree en las reuniones maravillosas y en las maravillosas "generaciones", que todavía preexisten en

\footnotetext{
${ }^{21}$ Hölderlin tiene un sentimiento místico hacia la vida.

22 Hiperion es la historia de un griego moderno que vive el sueño de infinita belleza y perfección de la Grecia antigua. Esta belleza la encuentra encarnada en una muchacha, Diótima, de la que se enamora. Pero abandona a ésta para hacer realidad su ideal de perfección espiritual y se lanza a la lucha para volver a encauzar a su patria hacia este ideal. Mas el resultado es la desconfianza y la desilusión. Entonces renuncia a su amada, se retira a la soledad, se nutre de su sueño y acaba por gozar y exaltar su propio dolor (Traducción Italiana, ed. Alfero, Turín, 1931).
} 
lo interno. Se trata de descubrir un mundo oculto para cuya estructura el lenguaje no tiene ningún nombre. La fantasía, el entendimiento, la razón, sólo son funciones limitadas y parciales; detrás de ellas como función fundamental se encuentra lo peculiarmente creador, es decir, la genialidad. Pero el genio no es la disposición excepcional del artista, sino el núcleo esencial de la naturaleza humana. El conocimiento verdadero arraiga en la revelación interior. Aquí es el punto en que Novalis transciende a Fichte en su doctrina de la intuición intelectual del yo. La aprehensión de sí mismo es un acto enigmático que descansa en una comunidad interior consigo mismo, es decir, en una conversación con uno mismo, o en un trato interior. Este acto da testimonio del misterio del alma. Frente al yo real se encuentra un "yo ideal". Para Novalis el hombre es un mago evocador de mundos, creador y aniquilador de la realidad. En su novela Heinrich von Hofterdingen, este soñador celebra con palabras entusiastas el poder infinito del hombre sobre el mundo. «El sentimiento moral, dice Nova$\mathrm{lis}^{23}$, es en nosotros el sentimiento de la potencia absoluta de crear, de la libertad productora, de la personalidad infinita del microcosmos». Este dilatarse del hombre hasta el infinito, este su transformarse en una voluntad infinita, creadora de la naturaleza y omnipotente, es el fundamento del idealismo mágico de Novalis $^{24}$

${ }^{23}$ En Schriften, ed. Heilborn, Jena, 1907, p. 375.

${ }^{24}$ Mago es quién sabe dominar la naturaleza hasta el punto de hacerla servir a los fines por él establecidos. 